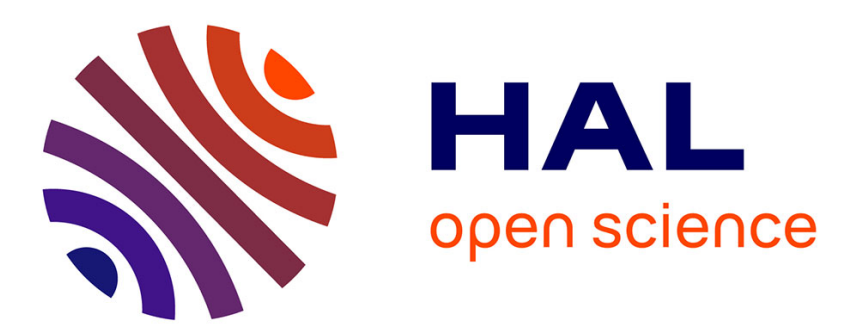

\title{
Semi-Conductor to Metal Transition in Liquid Selenium at High Pressure and High Temperature
}

Y. Soldo, J. Hazemann, D. Aberdam, E. Pernot, M. Inui, J. Jal, K. Tamura, J. Dupuy-Philon, D. Raoux

\section{- To cite this version:}

Y. Soldo, J. Hazemann, D. Aberdam, E. Pernot, M. Inui, et al.. Semi-Conductor to Metal Transition in Liquid Selenium at High Pressure and High Temperature. Journal de Physique IV Proceedings, 1997, 7 (C2), pp.C2-983-C2-985. 10.1051/jp4:19972108 • jpa-00255165

\section{HAL Id: jpa-00255165 https://hal.science/jpa-00255165}

Submitted on 1 Jan 1997

HAL is a multi-disciplinary open access archive for the deposit and dissemination of scientific research documents, whether they are published or not. The documents may come from teaching and research institutions in France or abroad, or from public or private research centers.
L'archive ouverte pluridisciplinaire HAL, est destinée au dépôt et à la diffusion de documents scientifiques de niveau recherche, publiés ou non, émanant des établissements d'enseignement et de recherche français ou étrangers, des laboratoires publics ou privés. 


\title{
Semi-Conductor to Metal Transition in Liquid Selenium at High Pressure and High Temperature
}

\author{
Y. Soldo*, J.L. Hazemann****, D. Aberdam*, E. Pernot**, M. Inui***, J.F. Jal****, K. Tamura***, \\ J. Dupuy-Philon**** and D. Raoux*
}

\author{
* Laboratoire de Cristallographie-CNRS, 25 avenue des Martyrs, BP. 166, 38042 Grenoble cedex 09, \\ France \\ ** LGIT CNRS, UJF, BP. 53X, 38041 Grenoble, France \\ *** Faculty of Integrated Arts and Sciences, Hiroshima University, Higashihiroshima 739, Japan \\ **** Département de Physique des Matériaux, UCB Lyon I, France
}

\begin{abstract}
Near its critical point $\left(385\right.$ bars, $1615^{\circ} \mathrm{C}$ ), semiconducting liquid Se gives way to a metallic state (SC-M transition). In this paper we report for the first time XANES and EXAFS measurements on liquid $\mathrm{Se}$ in the temperature and pressure range up to 600 bars and $1500^{\circ} \mathrm{C}$, in the temperature interval where the electrical conductivity shows the SC-M transition. Both the XANES spectra and the Fourier transforms of the EXAFS modulations present significant modifications. The results obtained with an anharmonic analysis (cumulant expansion method) show that the helical chains get shorter as temperature raises, but, even at the highest temperatures, they are not shorter than 5 atoms. The reconstruction of the distances distribution function for the first shell shows that, in the metallic region, the mean distance value decreases down to about $2.23 \AA$ and the distributions show an important asymmetry towards lower values of $R$.
\end{abstract}

\section{INTRODUCTION}

Crystalline trigonal selenium is a semiconductor. It consists of helical chains where each atom is covalently bonded to two neighbours. The twofold coordination in helical chains is largely preserved in the liquid phase near the melting point, Se being an example of a liquid semiconductor in which the chemical bond is purely covalent. The interest of studying liquid selenium is due to the fact that, near its critical point $\left(385 \mathrm{bars}, 1615^{\circ} \mathrm{C}\right)$, the semiconducting (SC) properties give way to a metallic (M) state. To understand the mechanism of the SC-M transition, it is necessary to access the structural parameters, however the information in this $\mathbf{P}-\mathrm{T}$ region has been quite limited because of technical difficulties. It has been speculated for long time that the metallic nature comes from threefold coordinated centres in branched chains which should be largely created in the high temperature and high pressure region. However recently Tamura et al. [1] made X-ray diffraction measurements up to $1500{ }^{\circ} \mathrm{C}$ and 800 bars and their results give no evidence for the existence of threefold coordinated centres.

The need of accurate information on the local structure moved us to make for the first time EXAFS measurements on liquid selenium in the temperature and pressure range up to 600 bars and $1500^{\circ} \mathrm{C}$. The measurements at high pressures and high temperatures, where the EXAFS signal is quite small, have been made possible by the availability of the high-brilliance X-ray source at the European Synchrotron Radiation Facility (ESRF) at Grenoble (France).

\section{EXPERIMENTAL CONDITIONS}

EXAFS measurements have been performed in transmission mode using the spectrometer installed on the BM32 beam-line at the ESRF. We used a sagitally focusing Si(111) double crystal monochromator, with an angular accuracy of $2 \cdot 10^{-4} \mathrm{mrad}$. The storage ring was operated in the multi-bunch mode at $200 \mathrm{~mA}$ and $6 \mathrm{GeV}$. The intensities of the incident, I0, and transmitted, I, beam were monitored through the measurement of the intensities scattered by a kapton foil. These measurements are performed using two $\mathrm{Si}$ diodes located upwards and below the beam. We registered EXAFS spectra for crystalline Se at room conditions and for liquid Se at 100 bars and $500^{\circ} \mathrm{C}$. Then we recorded a series of spectra at 600 bars and at various temperatures from $500^{\circ} \mathrm{C}$ to $1500^{\circ} \mathrm{C}$, which were chosen to cross the SC-M transition.

The experimental conditions of high pressure and high temperature were achieved using a vessel made of super-hightension steel, developed by Tamura et al. [2]. The vessel is pressurised by He gas, which has a low absorption in the energy range of the Se K edge. Its high purity grade (99.9999\%) minimises the risk of metal oxidation. The sample is located on the centre of the vessel and it is contained in a cell made in polycrystalline sapphire. Its thickness, $30 \mu \mathrm{m}$, has been chosen so that the jump at the absorption edge is about unity. It is heated by a heating element made of a Mo wire. The temperature is measured at three locations by B-type thermocouples which are in close contact with the sapphire walls enclosing the liquid sample.

\section{RESULTS}

XANES spectra exhibit a significant modification in their shape between $1000^{\circ} \mathrm{C}$ and $1250^{\circ} \mathrm{C}$, around which the $\mathrm{SC}-\mathrm{M}$ transition occurs, and they undergo an energy shift towards a lower energy by about $2 \mathrm{eV}$. These modifications are a clear signature of the SC-M transition.

The EXAFS oscillations are severely damped because of the substantial increase of the Debye-Waller factor, but, even at the highest temperatures, they are still clearly seen (see Fig. 1). By looking at the radial distribution functions (RDF) shown 
in Fig. 2 by the modulus of the Fourier transforms (FT) of $\mathrm{k}^{2} \chi(\mathrm{k})$, it is already possible to obtain some qualitative information about the SC-M transition.

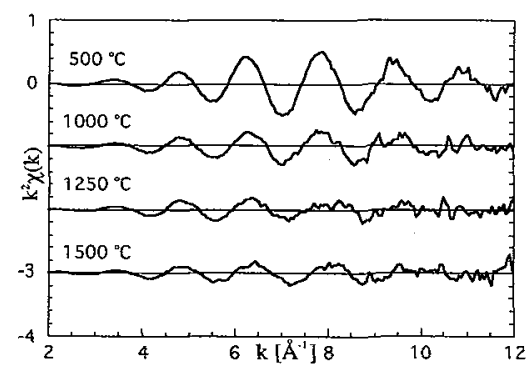

Figure 1: EXAFS oscillations at 600 bars and at different temperatures.

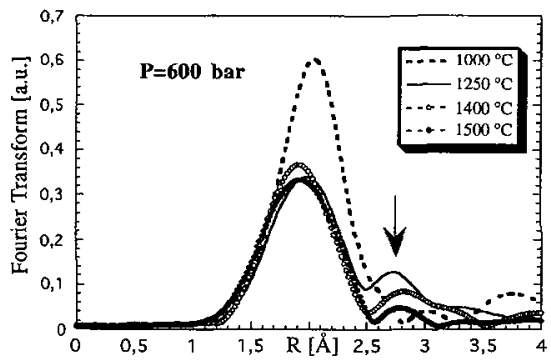

Figure 2: Modulus of the Fourier transform for liquid selenium at $1000^{\circ} \mathrm{C}$ (semiconductor state) and at $1250^{\circ} \mathrm{C}, 1400^{\circ} \mathrm{C}$ and $1500^{\circ} \mathrm{C}$ (metallic state).

Our reference sample, liquid Se at $500^{\circ} \mathrm{C}$ and 100 bars, shows a single peak, corresponding to the first neighbour interatomic distance at $2.33 \AA$ and to a coordination number of 2 . At 600 bars, and at higher temperatures, up to $1000^{\circ} \mathrm{C}$, all the FT's exhibit a single peak, the position of which does not significantly change. However at $1250^{\circ} \mathrm{C}$ two significant changes occur in the FT signal. The peak position shifts towards lower distances by about $0.05 \AA$ and a second shell clearly appears, as shown by the arrow in Fig. 2. Its position corresponds to an interatomic distance larger than the first one by about $0.7 \AA$. As temperature raises, the intensity of this second shell peak strongly decreases.

\section{DATA ANALYSIS BEYOND THE HARMONIC APPROXIMATION: CUMULANT EXPANSION}

Firstly we analysed our data using the well known single scattering, harmonic approximation to describe the EXAFS signal. In order to determine the structural parameters such as bond length $R$, coordination number $N$ and Debye Waller factor $\sigma$, we used a standard fitting procedure[3]. The results obtained in this approximation are given in Table 1. We note that the first shell coordination number decreases down to about 1 at $1500^{\circ} \mathrm{C}$, which is unrealistic in the liquid state and in disagreement with the diffraction results obtained by Tamura et al. [1]. Furthermore the values of the Debye Waller factor are quite large. These two points indicate that it is necessary to go beyond the harmonic approximation.

In the single scattering approximation, EXAFS does not sample the real RDF $\rho(R)$, but the so called "effective distribution" $P(R, \lambda)$, where $P(R, \lambda)=\rho(R) \exp (-2 R / \lambda) / R^{2}, \lambda$ being the mean free path of electrons. If $\rho(R)$ is not gaussian or not sufficiently narrow, the difference between real and effective RDF's is not negligible and the use of the standard formula can lead to significant errors. In this case it is useful to expand around $\mathrm{k}=0$ the Fourier transform of the effective RDF, which gives the EXAFS modulations, in a series of cumulants $C_{n}[4]$ :

$$
\int_{0}^{\infty} P(r, \lambda) e^{2 i k r} d r=\exp \left(\sum_{n=0}^{\infty} \frac{(2 i k)^{n}}{n !} C_{n}\right)
$$

$\mathrm{C}_{0}$ depends on the normalisation of the distribution, $\mathrm{C}_{1}$ and $\mathrm{C}_{2}$ correspond, respectively, to the mean value of the interatomic distance and its variance $\sigma^{2}$ averaged over the effective RDF. Higher order cumulants characterise the shape of the distribution; they are zero for gaussian distributions. It is possible to express the EXAFS function in terms of cumulants. Their values $\mathrm{C}_{n}$ and the coordination number $\mathrm{N}$ can be obtained fitting the difference of the phases and the ratio of the amplitudes of the 
unknown sample and of the reference one. Once the cumulants $C_{n}$ known, it is possible to reconstruct the effective and successively the real RDF.

Table 1 gives the structural parameters obtained using the harmonic approximation and the cumulant expansion till $\mathrm{C}_{6}$. All the data we will refer to, have been recorded at 600 bars. Due to the small intensity of the second shell contribution, we could analyse quantitatively only the first shell, though a two shell fitting has been used for data at $\mathrm{T} \geq 1250^{\circ} \mathrm{C}$ in the harmonic case to sort out the contributions of the second shell. At $1400^{\circ} \mathrm{C}$ and $1500^{\circ} \mathrm{C}$, the intensity of the second peak in the RDF is weak enough to be filtered in $\mathrm{R}$ space. However at $1250^{\circ} \mathrm{C}$ it is not the case and we have not been able to get reliable values of the cumulants.

At $500{ }^{\circ} \mathrm{C}$ we found that the value of the cumulants is negligible, so that there is no need to correct the results obtained within the harmonic analysis. However at higher temperatures the values of the cumulants become large. In all cases, we tested the convergence of the cumulant expansion using formula (1). We found that at $650^{\circ} \mathrm{C}$ the results obtained calculating the cumulants till $\mathrm{C}_{4}$ or till $\mathrm{C}_{6}$ are almost the same, while for $\mathrm{T} \geq 800^{\circ} \mathrm{C}$ it is necessary to calculate the cumulants till $\mathrm{C}_{6}$. So considering the anharmonic corrections for $\mathrm{T} \geq 650^{\circ} \mathrm{C}$, we find that $\mathrm{N}$ does not decrease below about 1.6, while $\sigma$ continuously increases up to $0.18 \AA$. We reconstructed the $\rho(R)$ distributions to get the mean values of the interatomic distances $R_{m}$ and the values $\delta$ of the asymmetry. As the harmonic analysis already suggested, the interatomic distance decreases by about $0.1 \AA$ At the SC-M transition. The distributions begin to be asymmetric at $650^{\circ} \mathrm{C}$, but it is only at higher temperatures that $\delta$ is largely negative, corresponding to an asymmetry towards lower values of $R$.

Table 1: Structural parameters obtained for the first shell in the harmonic approximation and using the cumulant expansion till $\mathrm{C}_{6}$. The interatomic distances $R_{m}$ are the mean values of the real RDF $\rho(R)$ calculated with the cumulants; $\delta$ is the asymmetry of $\rho(R), N$ is the coordination number and $\sigma$ is the Debye Waller factor. We could correctly estimate the error bars only in the harmonic approximation: we considered both the experimental statistical noise and the correlation between the extracted parameters.

\begin{tabular}{|c|c|c|c|c|c|c|c|}
\hline & \multicolumn{3}{|c|}{ HARMONIC APPROXIMATION } & \multicolumn{4}{c|}{ CUMULANT EXPANSION } \\
\hline $\mathbf{T}\left[{ }^{\circ} \mathbf{C}\right]$ & $\mathbf{N}$ & $\sigma \cdot \mathbf{1 0}^{-\mathbf{2}}[\AA \mathbf{\AA}]$ & $\mathbf{R}[\AA]$ & $\mathbf{N}$ & $\sigma \cdot \mathbf{1 0}^{-2}[\AA]$ & $\mathbf{R}_{\mathbf{m}}[\AA]$ & $\delta$ \\
\hline $\mathbf{5 0 0}$ & $1.91 \pm 0.12$ & $9.6 \pm 0.25$ & $2.327 \pm 0.003$ & \multicolumn{4}{c|}{ NO ANHARMONIC EFFECTS } \\
\hline $\mathbf{6 5 0}$ & $1.53 \pm 0.11$ & $9.3 \pm 0.3$ & $2.319 \pm 0.004$ & 1.74 & 10.3 & 2.35 & -0.048 \\
\hline $\mathbf{8 0 0}$ & $1.28 \pm 0.12$ & $9.1 \pm 0.4$ & $2.328 \pm 0.004$ & 1.91 & 13.1 & 2.35 & -0.167 \\
\hline $\mathbf{1 0 0 0}$ & $1.42 \pm 0.18$ & $11.2 \pm 0.5$ & $2.335 \pm 0.006$ & 1.67 & 13.1 & 2.32 & -0.143 \\
\hline $\mathbf{1 2 5 0}$ & $1.50 \pm 0.23$ & $13.3 \pm 0.6$ & $2.292 \pm 0.009$ & & NOTCALCULATED & \\
\hline $\mathbf{1 4 0 0}$ & $0.99 \pm 0.15$ & $11.6 \pm 0.6$ & $2.270 \pm 0.009$ & 1.79 & 18.1 & 2.22 & -0.200 \\
\hline $\mathbf{1 5 0 0}$ & $0.92 \pm 0.13$ & $11.8 \pm 0.6$ & $2.255 \pm 0.009$ & 1.58 & 17.8 & 2.23 & -0.167 \\
\hline
\end{tabular}

\section{DISCUSSION}

At the SC-M transition, XANES spectra clearly give evidence of an electronic/structural modification. In the same temperature range the FT shows the growth of a second shell at about $2.9 \AA$ and a shift of the first peak position towards lower values of $R$. The decrease of the first shell coordination number obtained using an anharmonic analysis (cumulant expansion method) shows that the helical chains get shorter as temperature raises. However even at the highest temperatures, they are at least 5 atoms long. The reconstruction of the real RDF for the first shell shows that the mean distance value decreases down to about $2.23 \AA$ at $1500^{\circ} \mathrm{C}$. As the metallic state is approached, the RDF's also show an important asymmetry towards lower values of R. Our results on the first shell RDF seem to agree with the model proposed by Tamura et al. [1], where the metallic behaviour of fluid $\mathrm{Se}$ is related to short chains with planar zig-zag conformation. But the lack of quantitative information about the second shell found in the metallic state doesn't allow to exclude the presence of threefold coordinated centers. Diffuse wide and small angle $\mathrm{X}$-ray scattering experiments are planned to get more reliable information about the medium range order and the presence of density fluctuations in the metallic region.

\section{References}

[1] K.Tamura and S. Hosokawa, Phys. Chem. 96 (1992) 681

[2] S.Hosokawa, K.Tamura, M.Inui, M.Yao, H.Endo and H. Hoshino, J.Chem.Phys. 97(2) (1992) 786

[3] B.K.Teo,EXAFS: Basic Principles and Data Analysis 1986 Berlin: Springer

[4] G.Dalba, P.Fornasini, F.Rocca, Phys. Rev. B 47 (1993) 8502 